\title{
Les comparaisons Interlaboratoires sont incontournables !
}

\author{
David LELONG, PSA Peugeot Citroën 78301 Poissy
}

Résumé. Les Comparaisons Inter laboratoires ont plusieurs objectifs et finalités dans de nombreux secteurs de l'industrie et s'appliquent pour tout processus de mesure fournissant une grandeur physique chiffrée ou non mais surtout exploitable ! Les Comparaisons Inter laboratoires permettent de :

- Déterminer une valeur à un matériau de référence,

- Caractériser des performances d'une méthode de mesure : justesse et fidélité

- Evaluer la performance d'un laboratoire, essais d'aptitude

- Qualifier et Habiliter le personnel

- Optimiser le raccordement métrologique des instruments de mesures.

- Surveiller un processus de mesure.

- Réaliser des comparaisons inter-instruments (un GT est actuellement en cours à l'AFNOR)

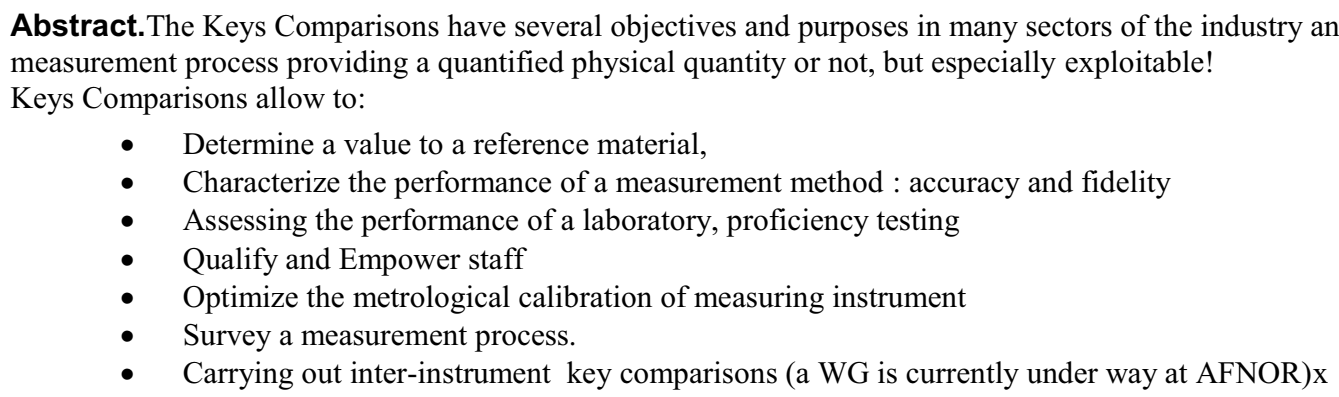

\section{Introduction}

La pratique des comparaisons entre laboratoires est un axe clé pour le contrôle des processus de mesure. Jusqu'à présent, les comparaisons inter laboratoires sont considérées comme les outils les plus efficaces afin d'évaluer la qualité globale des processus de mesure. L'évolution de l'organisation PSA dans une entreprise étendue exige que nos fournisseurs et prestataires de services appliquent le standard CIL et démontrent la qualité de leur processus de mesure.

Participer à des comparaisons entre laboratoires devient une nécessité pour l'entreprise afin de prouver la maitrise des processus mis en œuvre. La comparaison Inter laboratoires devient un juge de paix permettant de donner un niveau de confiance du laboratoire.

Les enjeux sont bien sur liés à la Qualité mais aussi économiques!

PSA organise des audits de laboratoires d'essais et des métrologies selon la norme de référence A109000 issue de la norme ISO17025.

Dans le cadre de ces audits A109000, nous vérifions la capacité des laboratoires de métrologie ou d'essais à maitriser leur processus de mesure. Les comparaisons inter laboratoires font partie des exigences incontournables du référentiel.

Aujourd'hui, nous suivons cette maitrise via les habilitations du personnel, et les essais qui font partie intégrante des compétences techniques de l'équipe et

valident l'aptitude des laboratoires. C'est aussi une méthode efficace pour estimer les incertitudes de mesure des processus de tests complexes.

Les résultats sont régulièrement présentés en rituel Qualité pour engager les plans d'action nécessaires.

PSA déploie cette culture au moyen d'une norme de référence et d'une formation pour amener au bon niveau la maturité du groupe.

Différents outils ont été mis en place pour fédérer la pratique et l'harmonisation des analyses. 


\section{Besoin d'accompagner et d'orienter les opérationnels}

Le métier expertise de la mesure accompagne les métiers de la mise en place à l'exploitation des résultats :

- $\quad$ Mise en place de la méthodologie

- Exploitation des calculs statistiques.

- Analyse et orientation des actions à engager

- Suivi et contrôle du solde des écarts

Tout cela dans une démarche de progrés permanent type PDCA.

\section{Synoptique sur l'accompagnement et le suivi des Laboratoires}

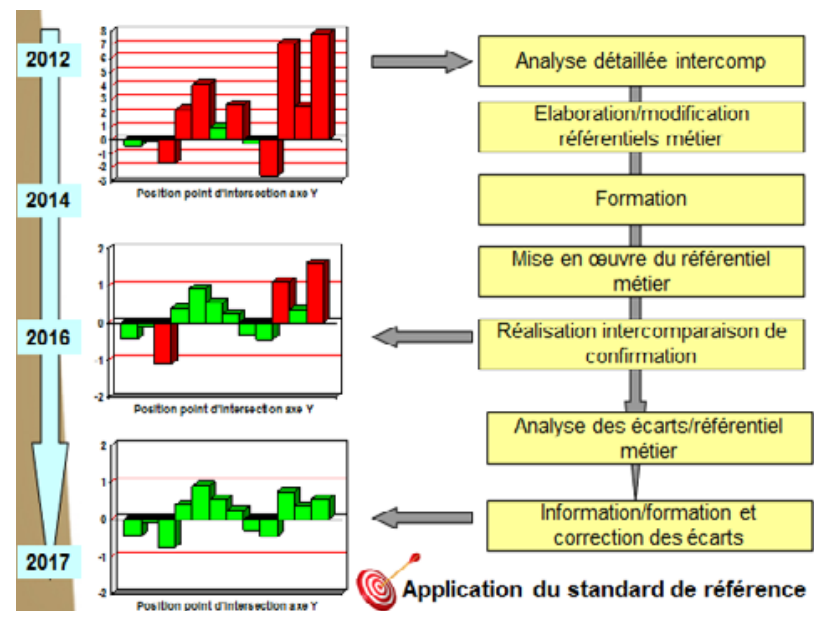

\section{Besoin d'un outil d'analyse et d'aide à la décision dans l'entreprise étendue}

Dans un contexte industriel d'entreprise étendue, PSA a besoin de s'appuyer sur un outil permettant de faire les calculs et graphiques associés ainsi qu'un rapport en automatique.. L'objectif est d'harmoniser la méthode d'évaluation et les critères au sein des différents métiers du groupe.

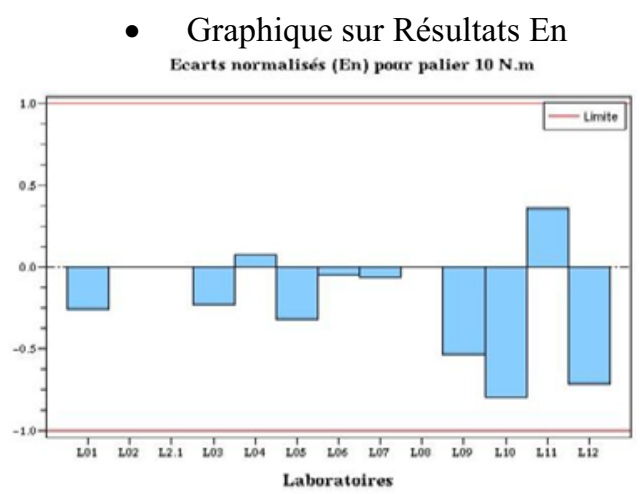

Graphique sur Résultats Erreur de justesse /Incertitudes

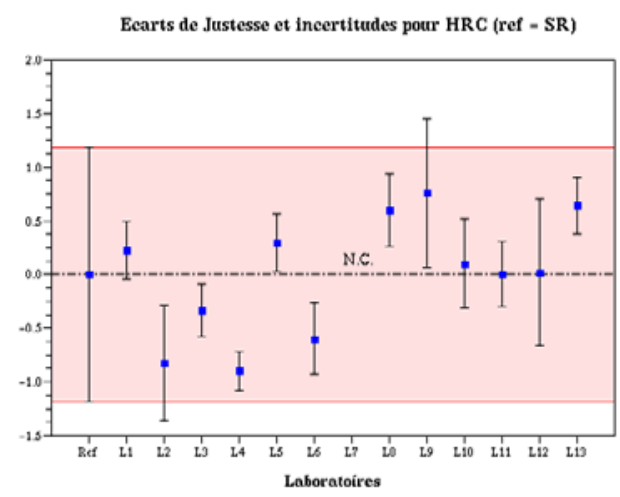

Dans le cadre des plans de progrès PSA Groupe des études sont en cours pour rechercher des solutions du marché afin de remplacer les outils actuels vieillissants.

Cet outil devra embarquer l'ensemble des besoins Mesures dont les Comparaisons Inter laboratoires :

- Outil intégrant CIL, Incertitudes, Acceptation des Processus Utilisation des modèles, Analyse des données

- Facilité de prise en main avec intégration des calculs statistiques (En , Grubbs , Cohran ,Monte Carlo ,....)

- Intégration Graphiques

- Edition de rapport en Automatique

Participer à des comparaisons inter laboratoires devient une nécessité pour l'entreprise afin de garantir la qualité des processus de mesure mis en œuvre aussi bien en interne qu'en externe. C'est devenu un incontournable reconnue au niveau international (cf ISO17025)!

Organiser les comparaisons au sein de son entreprise est une démarche pertinente mais n'est pas encore une pratique totalement répandue. 